\title{
Population pharmacokinetics of cefotaxime in intensive care patients
}

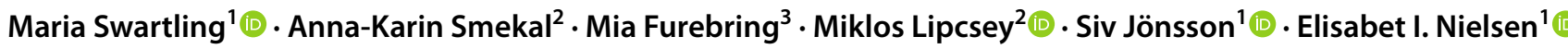

Received: 5 May 2021 / Accepted: 9 September 2021 / Published online: 1 October 2021

(c) The Author(s) 2021

\begin{abstract}
Purpose To characterise the pharmacokinetics and associated variability of cefotaxime in adult intensive care unit (ICU) patients and to assess the impact of patient covariates.

Methods This work was based on data from cefotaxime-treated patients included in the ACCIS (Antibiotic Concentrations in Critical Ill ICU Patients in Sweden) study. Clinical data from 51 patients at seven different ICUs in Sweden, given cefotaxime (1000-3000 mg given 2-6 times daily), were collected from the first day of treatment for up to three consecutive days. In total, 263 cefotaxime samples were included in the population pharmacokinetic analysis.

Results A two-compartment model with linear elimination, proportional residual error and inter-individual variability (IIV) on clearance and central volume of distribution best described the data. The typical individual was 64 years, with body weight at ICU admission of $92 \mathrm{~kg}$ and estimated creatinine clearance of $94 \mathrm{~mL} / \mathrm{min}$. The resulting typical value of clearance was $11.1 \mathrm{~L} / \mathrm{h}$, central volume of distribution $5.1 \mathrm{~L}$, peripheral volume of distribution $18.2 \mathrm{~L}$ and inter-compartmental clearance $14.5 \mathrm{~L} / \mathrm{h}$. The estimated creatinine clearance proved to be a significant covariate on clearance $(p<0.001)$, reducing IIV from 68 to $49 \%$.

Conclusion A population pharmacokinetic model was developed to describe cefotaxime pharmacokinetics and associated variability in adult ICU patients. The estimated creatinine clearance partly explained the IIV in cefotaxime clearance. However, the remaining unexplained IIV is high and suggests a need for dose individualisation using therapeutic drug monitoring where the developed model, after evaluation of predictive performance, may provide support.
\end{abstract}

Keywords Population pharmacokinetics $\cdot$ Modelling $\cdot$ Cefotaxime $\cdot$ Critically ill

Elisabet I. Nielsen

elisabet.nielsen@farmaci.uu.se

Maria Swartling

maria.swartling@farmaci.uu.se

Anna-Karin Smekal

anna-karin.smekal@surgsci.uu.se

Mia Furebring

mia.furebring@medsci.uu.se

Miklos Lipcsey

miklos.lipcsey@surgsci.uu.se

Siv Jönsson

siv.jonsson@farmaci.uu.se

1 Department of Pharmacy, Uppsala University, Uppsala, Sweden

2 Department of Surgical Sciences, Anaesthesiology and Intensive Care, Uppsala University, Uppsala, Sweden

3 Department of Medical Sciences, Infectious Medicine, Uppsala University, Uppsala, Sweden

\section{Introduction}

Infections are common in critically ill patients and associated with considerable morbidity, mortality and healthcare costs [1]. Cefotaxime is a broad-spectrum beta-lactam antibiotic used in the empirical treatment of several serious infections, and it is currently the most commonly used intravenous cephalosporin in Sweden according to national statistics on antibiotic use.

Cefotaxime disposition has previously been described [2] by a two-compartment model, with linear pharmacokinetics (PK). The half-life in healthy volunteers is reported to be around $1 \mathrm{~h}$, with $80 \%$ of an intravenous dose excreted in urine whereas $20 \%$ is recovered in faeces following biliary excretion. Cefotaxime is partially metabolised to desacetylcefotaxime, a metabolite with antibacterial activity reported to be two to 16 times less than that of cefotaxime depending on bacterial species [3]. 
Critically ill patients with sepsis frequently have significant physiological alterations that can lead to changed PK of the hydrophilic beta-lactams [4]. A large increase in volume of distribution $(V)$ has been observed, and this effect is especially pronounced in burn patients [5]. Further, critically ill patients are at risk for acute kidney injury and reduced clearance $(C L)$ of renally excreted antibiotics [4] as well as augmented renal clearance (ARC) [6].

The high inter-individual variability (IIV) of PK parameters in critically ill patients indicates the need for a more individualised approach to dosing. Population PK models describe the typical PK in a population as well as variability between individuals and may include covariates that help explain the variability [7]. Such models can be used before the start of treatment to predict a dosing regimen that maximises the chances of reaching PK-related treatment targets. They can also be used to obtain individual PK parameters based on measured drug concentrations, and subsequently for individual predictions and dose selection. In the case of cefotaxime, there are published population PK models for elderly patients [8] and paediatric patients [9-12], but to our knowledge, no such model for adult intensive care unit (ICU) patients has been published.

The aim of this study was to characterise the PK and associated variability of cefotaxime in adult ICU patients, and to assess the impact of patient covariates relevant for clinical practice, using a nonlinear mixed-effects modelling approach.

\section{Materials and methods}

\section{Study design}

The current analysis is a sub-study to ACCIS (Antibiotic Concentrations in Critical Ill ICU Patients in Sweden), a prospective observational multi-centre $\mathrm{PK}$ study conducted at seven ICUs, including one burn unit, in five Swedish hospitals between December 2015 and July 2017 (Trial ID ACTRN12616000167460). The Uppsala regional ethical review board approved the study (EPN Dnr 2015/135), and informed consent was obtained from the patient. The next of kin was consulted if the patient was unable to give consent. The study was performed in line with the principles of the Declaration of Helsinki and its revisions.

\section{Patient population}

Patients above the age of 18 years admitted to the ICUs and treated with cefotaxime for a proven or suspected infection were included in the study. Patients were included within $24 \mathrm{~h}$ from initiation of cefotaxime treatment. Pregnant patients and patients with treatment restrictions were excluded in the study. Additionally, patients on renal replacement therapy were excluded in this current analysis. Demographic and covariate data were obtained from the patient medical records.

\section{Dosing regimen and sampling}

The cefotaxime dose was chosen on discretion of the treating physician, where the recommended dose ranges from 1000 to $2000 \mathrm{mg}$ three times daily (t.i.d.) depending on the severity of the infection and $3000 \mathrm{mg}$ four times daily for meningitis. An additional dose in the middle of the first dose interval is recommended in sepsis according to the national guidelines by the Swedish Society of Infectious Diseases. Cefotaxime was administered intravenously as a short infusion over $5 \mathrm{~min}$. Blood samples for measurement of cefotaxime concentrations were collected twice during one dosing interval per day, at mid-point and just before next dose, from the first day of treatment for up to three consecutive days if cefotaxime treatment was maintained. Nurses registered the exact time points for blood sample collection and dose administration in a case report form.

\section{Cefotaxime analysis}

Samples were centrifuged for $7 \mathrm{~min}$ at $2400 \mathrm{~g}$ within $3 \mathrm{~h}$ of collection, and serum was stored at $-70{ }^{\circ} \mathrm{C}$ for later analysis. Total serum cefotaxime concentrations were determined by LC-MS/MS at the Department of Clinical Pharmacology, Karolinska University Hospital Huddinge, Stockholm, Sweden. The bioanalytical method was validated according to the European Medicines Agency Guideline on bioanalytical method validation and has been previously described for beta-lactams [13]. The range of quantification was $0.50-50 \mathrm{mg} / \mathrm{L}$ with a total coefficient of variation of $11.6 \%$ at the lower limit of quantification (LLOQ) and $\leq 6.0 \%$ in the quantification range. Accuracy was within -6.8 to $+4.4 \%$.

\section{Pharmacokinetic modelling}

A population PK model was developed using NONMEM (version 7.4, Icon Development Solutions, Hanover, MD, USA) [14] and Pearl-Speaks-NONMEM [15] to assist with the execution of models. The first-order conditional estimation method with interaction was used for parameter estimation. R version 3.5 (R Foundation for Statistical Computing, Vienna, Austria) was used for data management and the xpose4 package [15] for model diagnostics and evaluation.

For hierarchical models, the likelihood ratio test was used to evaluate statistical significance for the inclusion of additional parameters in the model, with the assumption that the difference in objective function value (OFV) between two hierarchical models is $\chi^{2}$-distributed. A 
reduction of OFV of 3.84 between two models with one parameter difference was considered statistically significant with a $p$-value of 0.05 , whereas a reduction of OFV of 10.83 was considered significant with a $p$-value of 0.001 . Model selection was further guided by basic goodnessof-fit plots and simulation-based prediction-corrected visual predictive checks (pcVPCs) [16]. A nonparametric bootstrap $(n=2000)$ was used to evaluate the stability and the confidence intervals (CIs) for the final parameter estimates. Further, maximum a posteriori (MAP) estimation was performed using the final model with observations in the dataset to evaluate the precision of the individual parameter estimates.

One- and two-compartment models with linear elimination were evaluated. To describe the variability between patients, IIV terms assuming log-normally distributed individual parameters were tested and included if statistically significant. Different residual error models were evaluated (additive, proportional and slope-intercept).

Only patient covariates that are readily available in the clinical setting were considered for testing to explain part of the random parameter variability. As body size is expected to influence PK parameters [17] and as increased weight due to treatment with resuscitation fluids might reflect $V$ in a critically ill patient, weight at ICU admission was added before other covariates were included as a time-independent mechanistic covariate in line with allometric principles [17]. As cefotaxime is renally cleared to a large part, estimated creatinine clearance $(e C L c r)$, derived from the CockcroftGault formula [18], was tested as a covariate on $C L$ as a time-dependent continuous covariate. As the PK parameters might change during the course of treatment, day of antibiotic treatment was tested as a time-dependent categorical covariate on volume parameters and $C L$. Further, as $V$ in burn patients might be different from that in other ICU patients [5], the burn unit patient subgroup was tested as a time-independent categorical covariate on volume parameters. Finally, Simplified Acute Physiology Score 3 (SAPS3) was tested as a continuous covariate in an attempt to evaluate non-renal factors on $C L$. A stepwise forward inclusion procedure was used $(p<0.05)$ to build the full model, and stepwise backward elimination $(p<0.001)$ to determine the final model.

Time-dependent continuous covariates were carried forward within individuals to the next observation, without interpolation between measurements. In case of missing data in the beginning of the time series, the first observation was carried backwards. Missing data for the time-independent covariate body weight at ICU admission (7 individuals) were imputed using linear regression with weight before ICU admission. Missing data for SAPS3 (2 individuals) were imputed using the population mean. Fifteen cefotaxime concentrations ( $6 \%$ of the total number, with no consecutive concentrations below LLOQ) from eight individuals were below LLOQ and were set to LLOQ/2 [19].

Continuous covariates were incorporated and evaluated in the model using different relationships (linear, piecewise linear, exponential or power). Linear and piecewise linear relationships were parameterised as Typical $C L=\theta_{\text {median }} \times[$ $\left.1+\theta_{\text {cov }} \times\left(X_{i}-X_{\text {median }}\right)\right]$, where $\theta_{\text {median }}$ is the typical $C L$ at the covariate median and $\theta_{\text {cov }}$ is the covariate effect, in this case as a fractional change per unit different from the population covariate median $\left(X_{\text {median }}\right) ; X_{i}$ is the covariate value for the individual. For the piecewise linear model, different slope parameters in different parts of the covariate range with a common breakpoint was estimated and different breakpoints were evaluated in a stepwise manner. Categorical covariates were incorporated as an estimated fractional change $\left[1+\theta_{\text {cov }}\right]$ in typical value from the reference category.

\section{Results}

\section{Patient characteristics and data}

Fifty-one ICU patients, 18 treated in a burn unit ICU and 33 in general ICU units, were included in the current study with a total of 263 cefotaxime samples and a median of 6 (range 2-6) samples per patient. Four samples were excluded from the original dataset due to reported erroneous high concentrations at a supposed trough time point. Table 1 shows a summary of the patient characteristics and collected data. Figure 1 illustrates the observed serum cefotaxime concentrations in relation to time after dose. The most common causes of infection were lower respiratory tract infections in $59 \%(30 / 51)$ of the patients followed by skin and soft tissue infections in 25\% (13/51) and urinary tract infections in $8 \%$ (4/51). The administered cefotaxime doses ranged from 1000 to $3000 \mathrm{mg}$ given 2-6 times daily, with the most common regimen being $1000 \mathrm{mg}$ t.i.d, followed by $2000 \mathrm{mg}$ t.i.d. An extra dose in the first dose interval was given in four cases.

\section{Pharmacokinetic modelling}

A two-compartment model with linear elimination from the central compartment and a proportional residual error model best described the data. IIV was possible to estimate for $C L$ and central volume of distribution $(V c)$, and was found to be highly positively correlated. Therefore, the same IIV term was used for $V c$ and $C L$, with a scaling factor for $V c$ to allow for different magnitudes of IIV (Table 2, Fig. 2).

The estimated $C L C r$ was included as a piecewise linear covariate on $C L$ with a fixed slope of zero at values above $120 \mathrm{~mL} / \mathrm{min}$, as the estimated slope approached zero. This equals truncating $e C L c r$ at high values, a strategy applied before [20] when using Cockcroft and Gault. The model fit 
Table 1 Overview of patient characteristics and observed data for included patients $(n=51)$

\begin{tabular}{ll}
\hline Characteristic & $\begin{array}{l}\text { Values } \\
\text { Median (IQR) [range] or number (\%) }\end{array}$ \\
\hline Time-independent variables & \\
Age (years) & $64(50-73)[23-90]$ \\
Female & $18(35 \%)$ \\
SAPS3 at admission to ICU & $55(47-63)[25-89]$ \\
Body weight before ICU admission $(\mathrm{kg})$ & $87(79-99)[55-123]$ \\
Body weight at ICU admission $(\mathrm{kg})$ & $92(81-102)[55-124]$ \\
Time-dependent variables & \\
$e C L c r(m L / m i n)$, treatment days $1-3$ & $94(65-138)[5-258]$ \\
Treatment day 1 & $85(52-133)[5-208]$ \\
Treatment day 2 & $97(65-130)[16-207]$ \\
Treatment day 3 & $105(80-159)[19-258]$ \\
Serum cefotaxime trough ${ }^{\mathrm{a}}$ concentration $(\mathrm{mg} / \mathrm{L})$, treatment days $1-3$ & $1.8(0.8-5.7)[0.25-72]$ \\
$\quad(n=112)$ & \\
Treatment day $1(n=42)$ & $2.6(0.9-7.5)[0.25-72]$ \\
Treatment day $2(n=43)$ & $2.2(0.8-4.4)[0.25-70]$ \\
Treatment day $3(n=27)$ & $1.4(0.7-2.8)[0.25-12.2]$ \\
\hline
\end{tabular}

$I Q R$, interquartile range; SAPS3, Simplified Acute Physiology Score 3; ICU, intensive care unit; eCLcr, estimated creatinine clearance, determined using the Cockcroft and Gault formula and body weight at ICU admission

${ }^{\text {a }}$ Trough defined as a sample drawn $\leq 1 \mathrm{~h}$ before next scheduled dose improved with a statistically significant drop in OFV of 31 $(p<0.001)$ and a reduction of the unexplained variability in $C L$ from 68 to $49 \%$. Figure 3 illustrates the covariate effect of $e C L \mathrm{cr}$ on the typical value of $C L$, where a $10 \mathrm{~mL} / \mathrm{min}$ change in $e C L c r$ results in a $0.74 \mathrm{~L} / \mathrm{h}$ change in $C L$ for $e C L c r$ values at or below $120 \mathrm{~mL} / \mathrm{min}$. The $y$-intercept, $4.2 \mathrm{~L} / \mathrm{h}$,

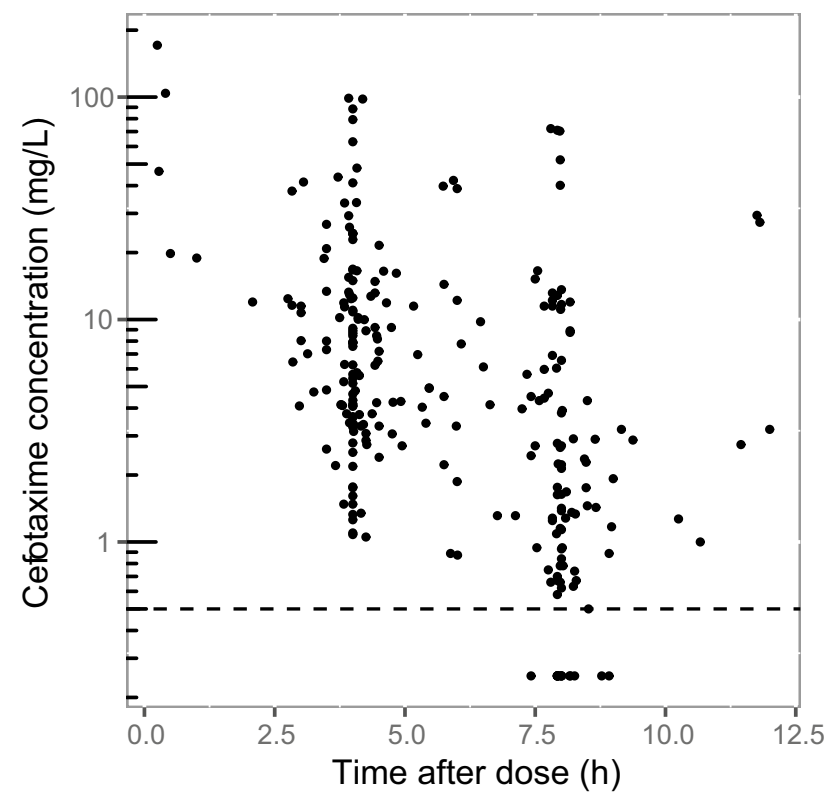

Fig. 1 A scatter plot of observed serum cefotaxime concentrations from 51 patients versus time after dose. Dots are the individual concentrations; dashed line marks the lower limit of quantification $(0.5 \mathrm{mg} / \mathrm{L})$ indicates the part of cefotaxime $C L$ that is not dependent on glomerular filtration represented by $e C L c r$ in this dataset. Inclusion of the other covariates (e.g. burn patients)

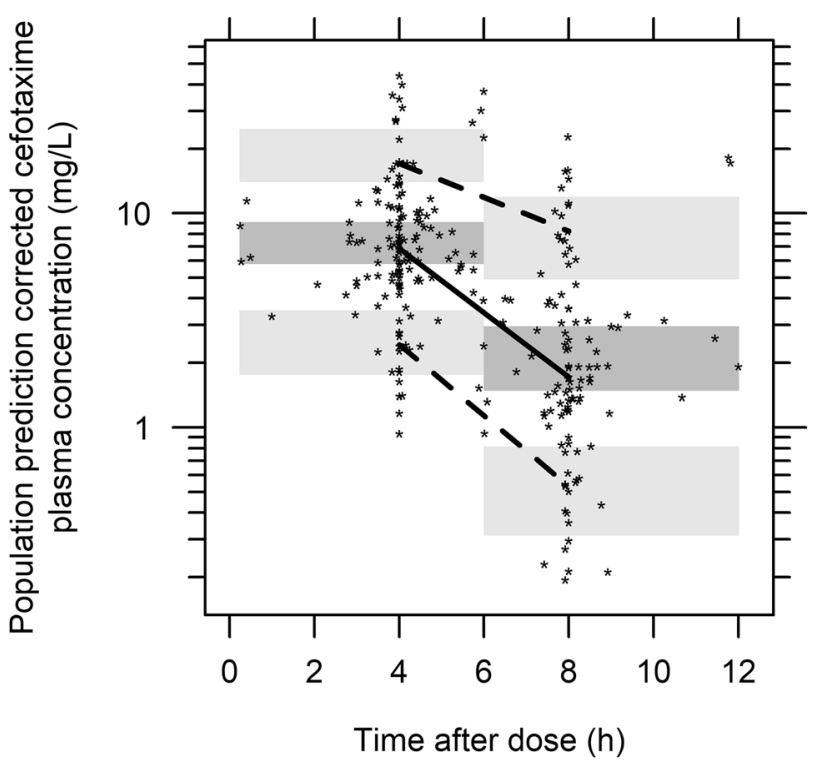

Fig. 2 Population prediction-corrected visual predictive check (pcVPC) for serum cefotaxime concentrations versus time after dose. Dots are the individual concentrations; solid line is the median of the observed data; dashed lines are the 10th and 90th percentiles of the observed data; shaded areas are the 95\% confidence intervals around the median (dark grey) and the 10th and 90th prediction intervals (light grey) of the simulated data 


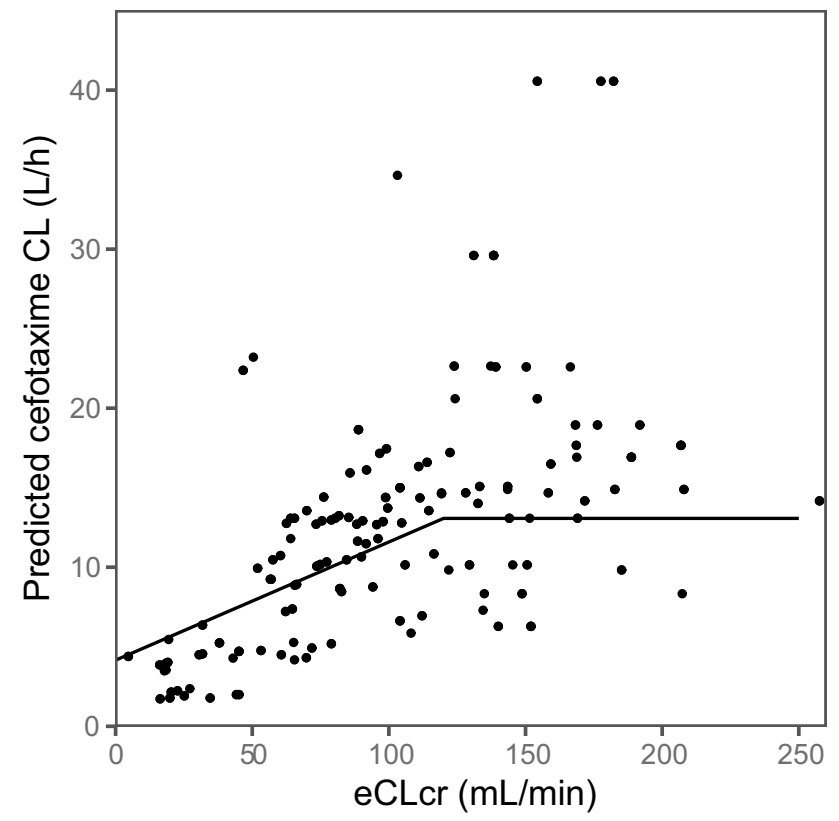

Fig. 3 Illustration of the covariate effect of estimated creatinine clearance $(e C L c r)$ on cefotaxime clearance $(C L)$. Dots are individual $C L$ data points; black lines mark the different linear change in the piecewise linear model with a common breakpoint at $e C L c r 120 \mathrm{~mL} / \mathrm{min}$

after inclusion of $e C L c r$ as a covariate did not significantly improve the model.

Table 2 shows the estimated parameters and associated uncertainty for the final PK model. Parameter estimates were provided with acceptable precision, and the residual diagnostics as well as the pcVPC (Fig. 2) demonstrate the model to be an adequate description of the data. The median relative standard error (RSE) from MAP estimation was $12 \%$ for $C L$ and $16 \%$ for $V c$ when using day 1 data (Online Resource 1, Table S1).

\section{Discussion}

Appropriate dosing of antibiotics in the early stage of sepsis and septic shock may be challenging due to the changing PK. Knowledge of the PK of antibiotics in the patient population is the first step towards further evaluation of target attainment and improvement of dosing recommendations. To address the lack of knowledge on cefotaxime PK in an ICU patient population, we developed a population PK model using data from cefotaxime treatment in ICU patients. Data collection started at the first day of cefotaxime treatment, thereby capturing the early treatment phase, and continued for up to three consecutive days. The study design included mid- and trough sampling of cefotaxime concentrations. Only four patients were on renal replacement therapy, and these patients were excluded from the analysis.

Our data show that cefotaxime follows a two-compartment PK model with linear elimination related to the individual $e C L c r$. These results are in line with previous descriptions of a biphasic disposition in adults [21, 22] and the findings
Table 2 Parameter estimates of the final population pharmacokinetic model, including NONMEM estimates with uncertainty, shrinkage and bootstrap median and confidence intervals $(n=2000)$

\begin{tabular}{llllll}
\hline Parameter & Parameter description & Estimate & \multicolumn{2}{l}{$(\mathrm{RSE} \%)$} & \multicolumn{2}{l}{ Bootstrap } \\
\cline { 5 - 6 } & & & {$[\mathrm{SHR} \%]$} & Median & $95 \%$ CI \\
\hline$C L(\mathrm{~L} / \mathrm{h})^{\mathrm{a}}$ & Clearance & 11.1 & $(8.2)$ & 10.9 & $8.5-14.0$ \\
$V c(\mathrm{~L})^{\mathrm{b}}$ & Central volume of distribution & 5.13 & $(28)$ & 4.84 & $1.5-17.6$ \\
$V p(\mathrm{~L})^{\mathrm{c}}$ & Peripheral volume of distribution & 18.2 & $(12)$ & 16.8 & $8.0-24.8$ \\
$Q(\mathrm{~L} / \mathrm{h})^{\mathrm{d}}$ & Inter-compartmental clearance & 14.5 & $(19)$ & 12.7 & $3.1-24.7$ \\
$\theta_{\text {cov }(e C L c r \leq 120)}$ & $e C L c r$ covariate effect on $C L$ & 6.67 & $(17)$ & 6.61 & $4.0-9.0$ \\
$f_{C L, V c}$ & Scaling factor for IIV $C L$ and $V c$ & 1.31 & $(39)$ & 1.30 & $0.2-2.5$ \\
IIV $C L(\% C V)$ & Inter-individual variability in $C L$ & 49 & $(15)[0.8]$ & 50 & $36-71$ \\
IIV $V c(\% C V)$ & Inter-individual variability in $V c$ & $64^{\mathrm{b}}$ & & & \\
Proportional & Proportional residual error & 33.3 & $(5.9)[7.8]$ & 32.9 & $29-37$ \\
$\quad$ residual error & & & & & \\
$(\% C V)$ & & & & & \\
\hline
\end{tabular}

RSE relative standard error, SHR shrinkage on standard deviation scale, CI confidence interval, $\theta$ population parameter, $e C L c r$ estimated creatinine clearance $(\mathrm{mL} / \mathrm{min})$, IIV inter-individual variability, $C V$ coefficient of variation, $B W$ body weight at intensive care unit admission, $C L_{i}$ individual $C L, V c_{i}$ individual $V c$, $V p_{i}$ individual $V p, Q_{i}$ individual $Q, \eta 1$ the deviation from the typical parameter in the individual

${ }^{\mathrm{a}} C L_{i}=\left(C L \times(B W / 92)^{0.75} \times\left(1+\theta_{\operatorname{cov}(e C L c r \leq 120)} \times(e C L c r-94) / 1000\right)\right) \times e^{\eta 1}$,

If $e C L c r>120 \mathrm{~mL} / \mathrm{min}: C L_{i}=\left(C L \times(B W / 92)^{0.75} \times\left(1+\theta_{\operatorname{cov}(e C L c r \leq 120)} \times(120-94) / 1000\right)\right) \times e^{\eta 1}$

${ }^{\mathrm{b}} V c_{i}=(V c \times(B W / 92)) \times e^{\eta 1 \times f C L, V c}$, the $C V$ for $V c$ was derived as $C V$ for CL times the estimated $f_{C L, V c}$

${ }^{\mathrm{c}} V p_{i}=V p \times(B W / 92)$

${ }^{\mathrm{d}} Q_{i}=Q \times(B W / 92)^{0.75}$ 
of Urien et al. [8], where a two-compartment model with $C L$ influenced by weight, plasma creatinine and age (all parts of the $e C L c r$ component) best described the data in elderly patients. That study also found serum protein concentrations to be a significant covariate. However, as this is not routinely registered in clinical practice in the current ICU setting, it was not evaluated in the present study.

The resulting cefotaxime typical $C L$ value $(11.1 \mathrm{~L} / \mathrm{h}$ for a subject with ICU weight of $92 \mathrm{~kg}$ and with $e C L c r$ of $94 \mathrm{~mL} / \mathrm{min}$ ) is in line with previously reported $10-13 \mathrm{~L} / \mathrm{h}$ in patients with liver disease [23] and higher than $5.4 \mathrm{~L} / \mathrm{h}$ in elderly patients [8]. Clearance of $15-17 \mathrm{~L} / \mathrm{h}$ in healthy adult populations $[21,22]$ is higher than in this ICU population as expected due to acute kidney injury in severe illness despite occasional cases of ARC.

Comparison of $V$ for the central compartment $(5.1 \mathrm{~L})$ and the peripheral compartment $(18.2 \mathrm{~L})$ with healthy adults $(6.5$ L resp. 8.6 L) [21] and elderly patients (5.5 L resp. $5.8 \mathrm{~L}$ ) [8] show a high peripheral volume of distribution $(V p)$ for the typical ICU patient in this study. However, higher total $V$ of 26-32 $\mathrm{L}$ has been reported for patients with liver disease in different disease stages [23]. Koedjik et al. [24] showed a median total $V$ in ICU patients on renal replacement therapy of $22.5 \mathrm{~L}$, results that are in line with this current investigation. High $V$ in ICU patients can be attributed to fluid leakage into the interstitial space and the use of resuscitation fluids [4], an effect that could be further pronounced by the high proportion of burn patients (35\%) in this study.

The intention of this study was to create a population PK model that reflects the clinical reality and includes covariates relevant for further use in a clinical setting. The treating physicians did the dose selection, and nurses on the wards did the cefotaxime sampling, but with a controlled registration of dosing and sampling times using case report forms. Only cefotaxime, and not its metabolite, was measured in this study. This is also according to clinical practice, since the European Committee on Antimicrobial Susceptibility Testing (EUCAST) clinical breakpoint [25] only considers the parent drug in setting the breakpoint to guide clinical dosing.

The estimated $C L c r$ has a modest effect on explaining the IIV in $C L$ in this model. Further, at low $e C L c r$, the covariate effect of $e C L c r$ on $C L$ is not fully captured despite testing of different covariate and residual error models. One reason for this can be that creatinine concentrations, routinely drawn at 6 a.m. each day with occasional extra samples, were used in the modelling process. This resulted in a potential gap between the sampled cefotaxime concentrations and corresponding creatinine of up to $24 \mathrm{~h}$, something that can be misleading in cases of rapid reduction of kidney function. Using a creatinine concentration from the next day and carrying the values backward could be an alternative but would then limit the use of the model for dose selection in clinic where one depends on available data before dosing. Further, even though creatinine is frequently used, it is well known that it is a poor marker in ICU patients with unstable kidney function [26]. Also, renal failure might be associated with multiple organ failure and affect non-renal components of $C L$. In an attempt to capture this, SAPS3 was tested as a potential covariate but did not improve the model further.

This analysis estimated a high unexplained variability in $V c$. We could not find covariates to explain the variability in the final model, although day of treatment was a statistically significant covariate $(p<0.05)$ before the inclusion of $e C L c r$ as a covariate on $C L$. There was no clear correlation between day of treatment and eCLcr $(r=0.17)$ that could help explain this. However, one reason might be lack of data for day 3 where only 34 out of the 51 patients had samples drawn due to discontinued treatment. In addition, a study period of 3 days might be too short to capture the clinical improvement and changes in $V$ over time in this relatively small and heterogeneous study population.

A limitation of the study is that the study design only included mid- and trough samples, potentially resulting in that $V c$ (RSE 28\%), $V p$ (RSE 12\%) and/or IIV for $V c$ (IIV scaling factor RSE 39\%) might not have been properly characterised. A model based on data with sampling earlier in the dosing interval could help describe $V$ in more detail.

The final model results in large unexplained IIV in $C L(49 \%)$ and $V c$ (64\%). Even if IIV could potentially be reduced by the successful inclusion of more covariates, it is likely that the unexplained variability between individuals in this population will remain high and that there is a need for individualisation of cefotaxime dosing.

A limitation of this study is that external validation has not been performed. Also, the model was developed using mainly mid- and trough samples and model performance has only been evaluated for this sampling strategy. However, after evaluation of predictive performance in the local setting, this model may provide support for model-based therapeutic drug monitoring and the implementation of dose individualisation in critically ill patients.

\section{Conclusion}

A population PK model was developed to describe cefotaxime PK and associated variability in adult ICU patients. Estimated creatinine clearance as a covariate partly explained the IIV in cefotaxime $C L$. However, the remaining unexplained IIV in $C L$ and $V c$ is still high and suggests a need for dose individualisation using therapeutic drug monitoring in critically ill where the developed model, after evaluation of predictive performance, may provide support.

Supplementary Information The online version contains supplementary material available at https://doi.org/10.1007/s00228-021-03218-6. 
Author contribution AS, ML and MF participated in the design of the clinical study and data acquisition. MS, EN and SJ participated in the current study conception and design. MS performed the data analysis supervised by EN and SJ. MS drafted the manuscript under the supervision of EN and SJ, and all other authors critically revised the manuscript. All authors read and approved the final version to be published.

Funding Open access funding provided by Uppsala University. The ACCIS study was funded by Regional Research Council Mid Sweden, grant nos. RFR-470321 and RFR-560491, as well as by the Region Uppsala research fund and the Olinder-Nielsen Family Fund for Research in Infectious Diseases. The pharmacometric analysis was in part supported by the VINNOVA-funded project PLATINEA (2018-03340).

Data availability The datasets analysed during the current study are available from the corresponding author on reasonable request.

Code availability The final model code describing the final model with input, compartments, equations, initial estimates for parameters and variances as well as estimation method is included in this published article (Online Resource 2).

\section{Declarations}

Ethical approval The current analysis is based on data from the ACCIS (Antibiotic Concentrations in Critical ill ICU patients Sweden) study approved by the Uppsala regional ethical review board (EPN Dnr 2015/135). The study was performed in line with the principles of the Declaration of Helsinki and its revisions.

Consent to participate Informed consent in the ACCIS study was obtained from the patient. The next of kin was consulted if the patient was unable to give consent.

Conflict of interest The authors declare no competing interests.

Open Access This article is licensed under a Creative Commons Attribution 4.0 International License, which permits use, sharing, adaptation, distribution and reproduction in any medium or format, as long as you give appropriate credit to the original author(s) and the source, provide a link to the Creative Commons licence, and indicate if changes were made. The images or other third party material in this article are included in the article's Creative Commons licence, unless indicated otherwise in a credit line to the material. If material is not included in the article's Creative Commons licence and your intended use is not permitted by statutory regulation or exceeds the permitted use, you will need to obtain permission directly from the copyright holder. To view a copy of this licence, visit http://creativecommons.org/licenses/by/4.0/.

\section{References}

1. Vincent JL, Rello J, Marshall J, Silva E et al (2009) International study of the prevalence and outcomes of infection in intensive care units. JAMA 302(21):2323-2329. https://doi.org/10.1001/jama. 2009.1754

2. Todd PA, Brogden RN (1990) Cefotaxime. An update of its pharmacology and therapeutic use. Drugs 40 (4): 608-651. https://doi. org/10.2165/00003495-199040040-00008

3. Brogden RN, Spencer CM (1997) Cefotaxime. A reappraisal of its antibacterial activity and pharmacokinetic properties, and a review of its therapeutic efficacy when administered twice daily for the treatment of mild to moderate infections. Drugs 53 (3): 483-510. https://doi.org/10.2165/00003495-199753030-00009

4. Roberts JA, Abdul-Aziz MH, Lipman J, Mouton JW et al (2014) Individualised antibiotic dosing for patients who are critically ill: challenges and potential solutions. Lancet Infect Dis 14(6):498 509. https://doi.org/10.1016/s1473-3099(14)70036-2

5. Udy AA, Roberts JA, Lipman J, Blot S (2018) The effects of major burn related pathophysiological changes on the pharmacokinetics and pharmacodynamics of drug use: an appraisal utilizing antibiotics. Adv Drug Deliv Rev 123:65-74. https://doi.org/10.1016/j. addr.2017.09.019

6. Udy AA, Roberts JA, Boots RJ, Paterson DL et al (2010) Augmented renal clearance: implications for antibacterial dosing in the critically ill. Clin Pharmacokinet 49(1):1-16. https://doi.org/ 10.2165/11318140-000000000-00000

7. Wicha SG, Märtson AG, Nielsen EI, Koch BCP et al (2021) From therapeutic drug monitoring to model-informed precision dosing for antibiotics. Clin Pharmacol Ther 109(4):928-941. https://doi. org/10.1002/cpt.2202

8. Urien S, Laurent N, Barre J, Druguet M et al (2004) Pharmacokinetic modelling of cefotaxime and desacetylcefotaxime-a population study in 25 elderly patients. Eur J Clin Pharmacol 60(1):11-16. https://doi.org/10.1007/s00228-003-0725-9

9. Maksoud E, Koehl B, Facchin A, Ha P et al (2018) Population pharmacokinetics of cefotaxime and dosage recommendations in children with sickle cell disease. Antimicrob Agents Chemother 62 (4). https://doi.org/10.1128/aac.00637-17

10. Béranger A, Oualha M, Urien S, Genuini M et al (2018) Population pharmacokinetic model to optimize cefotaxime dosing regimen in critically ill children. Clin Pharmacokinet 57(7):867-875. https://doi.org/10.1007/s40262-017-0602-9

11. Leroux S, Roué JM, Gouyon JB, Biran V et al (2016) A population and developmental pharmacokinetic analysis to evaluate and optimize cefotaxime dosing regimen in neonates and young infants. Antimicrob Agents Chemother 60(11):6626-6634. https://doi.org/ 10.1128/aac.01045-16

12. Ahsman MJ, Wildschut ED, Tibboel D, Mathot RA (2010) Pharmacokinetics of cefotaxime and desacetylcefotaxime in infants during extracorporeal membrane oxygenation. Antimicrob Agents Chemother 54(5):1734-1741. https://doi.org/10.1128/ aac.01696-09

13. Petersson J, Giske CG, Eliasson E (2016) Standard dosing of piperacillin and meropenem fail to achieve adequate plasma concentrations in ICU patients. Acta Anaesthesiol Scand 60(10):1425-1436. https://doi.org/10.1111/aas.12808

14. Beal SL SL, Boeckmann AJ, and Bauer RJ (Ed.) (2018) NONMEM 7.4 users guides. ICON plc, Gaithersburg, MD

15. Keizer RJ, Karlsson MO, Hooker A (2013) Modeling and simulation workbench for NONMEM: tutorial on Pirana, PsN, and Xpose. CPT: pharmacometrics \& systems pharmacology 2 (6): e50. https://doi.org/10.1038/psp.2013.24

16. Bergstrand M, Hooker AC, Wallin JE, Karlsson MO (2011) Prediction-corrected visual predictive checks for diagnosing nonlinear mixed-effects models. Aaps j 13(2):143-151. https:// doi.org/10.1208/s12248-011-9255-z

17. Anderson BJ, Holford NH (2008) Mechanism-based concepts of size and maturity in pharmacokinetics. Annu Rev Pharmacol Toxicol 48:303-332. https://doi.org/10.1146/annurev.pharmtox. 48.113006.094708

18. Cockcroft DW, Gault MH (1976) Prediction of creatinine clearance from serum creatinine. Nephron 16(1):31-41. https://doi.org/ $10.1159 / 000180580$

19. Beal SL (2001) Ways to fit a PK model with some data below the quantification limit. J Pharmacokinet Pharmacodyn 28(5):481504. https://doi.org/10.1023/a:1012299115260 
20. Goti V, Chaturvedula A, Fossler MJ, Mok S et al (2018) Hospitalized patients with and without hemodialysis have markedly different vancomycin pharmacokinetics: a population pharmacokinetic model-based analysis. Ther Drug Monit 40(2):212-221. https:// doi.org/10.1097/ftd.0000000000000490

21. Nix DE, Wilton JH, Hyatt J, Thomas J et al (1997) Pharmacodynamic modeling of the in vivo interaction between cefotaxime and ofloxacin by using serum ultrafiltrate inhibitory titers. Antimicrob Agents Chemother 41(5):1108-1114

22. Vallee F, LeBel M (1991) Comparative study of pharmacokinetics and serum bactericidal activity of ceftizoxime and cefotaxime. Antimicrob Agents Chemother 35(10):2057-2064. https://doi.org/ 10.1128/aac.35.10.2057

23. Ko RJ, Sattler FR, Nichols S, Akriviadis E et al (1991) Pharmacokinetics of cefotaxime and desacetylcefotaxime in patients with liver disease. Antimicrob Agents Chemother 35(7):1376-1380. https://doi.org/10.1128/aac.35.7.1376

24. Koedijk JB, Valk-Swinkels CG, Rijpstra TA, Touw DJ et al (2016) Pilot study of the pharmacokinetics of cefotaxime in critically ill patients with acute kidney injury treated with continuous renal replacement therapy. Antimicrob Agents Chemother 60(6):35873590. https://doi.org/10.1128/aac.02888-15

25. EUCAST (2020) Breakpoint tables for interpretation of MICs and zone diameters. Version 10.0. http://www.eucast.org. Accessed 15 June 2020

26. Bragadottir G, Redfors B, Ricksten SE (2013) Assessing glomerular filtration rate (GFR) in critically ill patients with acute kidney injury-true GFR versus urinary creatinine clearance and estimating equations. Crit Care 17(3):R108. https://doi.org/10. $1186 / \mathrm{cc} 12777$

Publisher's Note Springer Nature remains neutral with regard to jurisdictional claims in published maps and institutional affiliations. 